PAPER • OPEN ACCESS

Charged-current inclusive neutrino cross sections in the SuperScaling model

To cite this article: M.V. Ivanov et al 2016 J. Phys.: Conf. Ser. 724012020

View the article online for updates and enhancements.
Related content

Graphical imaging system for shifted spectrum in daughter nuclei of chargedcurrent v-nucleus reactions

J Sinatkas, T S Kosmas and V Tsakstara

- Charged-current inclusive neutrino cross

sections in the superscaling model

including quasielastic, pion production and

meson-exchange contributions

M V Ivanov, G D Megias, R GonzálezJiménez et al.

- Introduction to MiniBooNE and V chargedcurrent quasi-elastic results 


\title{
Charged-current inclusive neutrino cross sections in the SuperScaling model
}

\author{
M.V. Ivanov ${ }^{1,2}$, G.D. Megias ${ }^{3}$, R. González-Jiménez ${ }^{4}$, O. Moreno ${ }^{5}$, \\ M.B. Barbaro ${ }^{6}$, J.A. Caballero ${ }^{3}$, T.W. Donnelly ${ }^{5}$, A.N. Antonov ${ }^{1}$, \\ E. Moya de Guerra ${ }^{2}$, and J.M. Udías ${ }^{2}$ \\ ${ }^{1}$ Institute for Nuclear Research and Nuclear Energy, Bulgarian Academy of Sciences, Sofia \\ 1784, Bulgaria \\ ${ }^{2}$ Grupo de Física Nuclear, Departamento de Física Atómica, Molecular y Nuclear, Facultad \\ de Ciencias Físicas, Universidad Complutense de Madrid, Madrid E-28040, Spain \\ ${ }^{3}$ Departamento de Física Atómica, Molecular y Nuclear, Universidad de Sevilla, 41080 Sevilla, \\ Spain \\ ${ }^{4}$ Department of Physics and Astronomy, Ghent University, Proeftuinstraat 86, B-9000 Gent, \\ Belgium \\ ${ }^{5}$ Center for Theoretical Physics, Laboratory for Nuclear Science and Department of Physics, \\ Massachusetts Institute of Technology, Cambridge, Massachusetts 02139, USA \\ ${ }^{6}$ Dipartimento di Fisica, Università di Torino and INFN, Sezione di Torino, Via P. Giuria 1, \\ 10125 Torino, Italy \\ E-mail: martin.inrne@gmail.com
}

\begin{abstract}
SuperScaling model (SuSA) predictions to neutrino-induced charged-current $\pi^{+}$ production in the $\Delta$-resonance region are explored under MiniBooNE experimental conditions. The SuSA charged-current $\pi^{+}$results are in good agreement with data on neutrino flux-averaged double-differential cross sections. The SuSA model for quasielastic scattering and its extension to the pion production region are used for predictions of charged-current inclusive neutrinonucleus cross sections. The contribution of two-particle-two-hole vector meson-exchange current excitations is also considered within a fully relativistic model. Results are compared with the T2K experimental data for inclusive scattering.
\end{abstract}

\section{Introduction}

The analysis of neutrino oscillations is at present one of the main research topics in Physics. This explains the huge activity in the field and the numerous experiments that have been proposed in several facilities and covering a very wide range in energy. For the first time, experimental data on neutrino interactions are being measured with a high statistics and small error bands. As a consequence, a great variety of studies on the theoretical side have also appeared trying to describe the data. These include analyses from low-to-intermediate energies where the nuclear effects may play an important role, to very high energies where the nucleonic and/or subnucleonic degrees of freedom are the principal ingredients. In all cases, the understanding of neutrino properties is a key ingredient in elementary particle physics.

In most neutrino experiments, the interactions of the neutrinos occur with nucleons bound in nuclei. Model predictions for these reactions involve many different effects such as nuclear correlations, interactions in the final state, possible modification of the nucleon properties inside 
the nuclear medium, that presently cannot be computed in an unambiguous and precise way. This is particularly true for the channels where neutrino interactions take place by means of excitation of a nucleon resonance and ulterior production of mesons. The data on neutrinoinduced charged-current (CC) charged pion production cross sections on mineral oil recently released by the MiniBooNE collaboration [1] provide an unprecedented opportunity to carry out a systematic study of double differential cross section of the processes: $\nu_{\mu} p \rightarrow \mu^{-} p \pi^{+}$and $\nu_{\mu} n \rightarrow \mu^{-} n \pi^{+}$averaged over the neutrino flux.

New measurements of inclusive charged-current (CC) neutrino-nucleus scattering cross sections, where only the outgoing lepton is detected, have been recently performed by the T2K [2] collaboration. For neutrino energies around $1 \mathrm{GeV}(\mathrm{T} 2 \mathrm{~K})$ the main contributions to the cross sections are associated with quasielastic (QE) scattering and one pion $(1 \pi)$ production. In the present work we evaluate the CC neutrino inclusive cross sections within the SuperScaling approach (SuSA), introduced in [3] to describe neutrino-nucleus scattering by using electron scattering data instead of relying on specific nuclear models. Since meson-exchange currents (MEC) are known to violate superscaling, their contribution must be added to the superscaling result: this will be accomplished by using a parametrization [4] of the relativistic calculation of [5]. The parametrization is necessary in order to reduce the computation time, since the exact calculation involves 7-dimensional integrals.

\section{The SuSA model and charged-current neutrino-nucleus cross section}

\subsection{The SuSA scaling function in the $Q E$ - and $\Delta$-region}

One way of avoiding model-dependencies is to use the nuclear response to other leptonic probes, such as electrons, under similar conditions to the neutrino experiments. The analyses of the world data on inclusive electron-nucleus scattering $[6,7]$ confirmed the observation of superscaling and thus justified the extraction of a universal nuclear response to be also used for weak interacting probes. The present study uses the results found from studies superscaling (based on $\psi$-scaling variable) obtained from analyses of inclusive electron scattering data. The latter consists in constructing a "superscaling function" $f(\psi)$ obtained by removing the single-nucleon content from the double-differential cross section and plotting it versus a scaling variable $\psi(q, \omega)$. Scaling of the first kind of scaling function [i.e., no explicit $q$ dependence of $f(\psi)$ ] can be seen at excitation energies below the QE peak. Scaling of second kind [i.e., no dependence of $f(\psi)$ on the mass number] turns out to be excellent in the same region. When scaling of both the first and second type occurs, one says that superscaling has taken place. However, while there is a number of theoretical models that exhibit superscaling, such as for instance the relativistic Fermi gas (RFG) $[8,9]$, the nuclear response departs from the one derived from the experimental data. This showed the necessity to consider more complex dynamical pictures of finite nuclear systems - beyond the RFG - in order to describe the nuclear response at intermediate energies. SuSA predictions are based on the phenomenological superscaling function extracted from the world data on quasielastic electron scattering [10]. In figure 1 we present the quasielastic SuSA scaling function (phenomenological parametrization of the experimental data taken from [3]) in comparison with the RFG scaling function: $f_{\mathrm{RFG}}^{\mathrm{QE}}\left(\psi_{\mathrm{QE}}\right)=\frac{3}{4}\left(1-\psi_{\mathrm{QE}}^{2}\right) \theta\left(1-\psi_{\mathrm{QE}}^{2}\right)$, where $\psi_{\mathrm{QE}}$ is the $\mathrm{QE}$ scaling variable.

The SuSA model has been extended to the $\Delta$-resonance region [3] where the response of the nuclear system proceeds through excitation of internal nucleonic degrees of freedom. Indeed, a non-quasielastic cross section for the excitation region in which nucleon excitations, particularly the $\Delta$, play a major role was obtained by subtracting from the data QE-equivalent cross sections given by SuSA $[11,12]$. This procedure has been possible due to the large amount of available high-quality data of inelastic electron scattering cross sections on ${ }^{12} \mathrm{C}$, including also separate information on the longitudinal and transverse responses, the latter containing important contributions introduced by effects beyond the impulse approximation (non-nucleonic). 


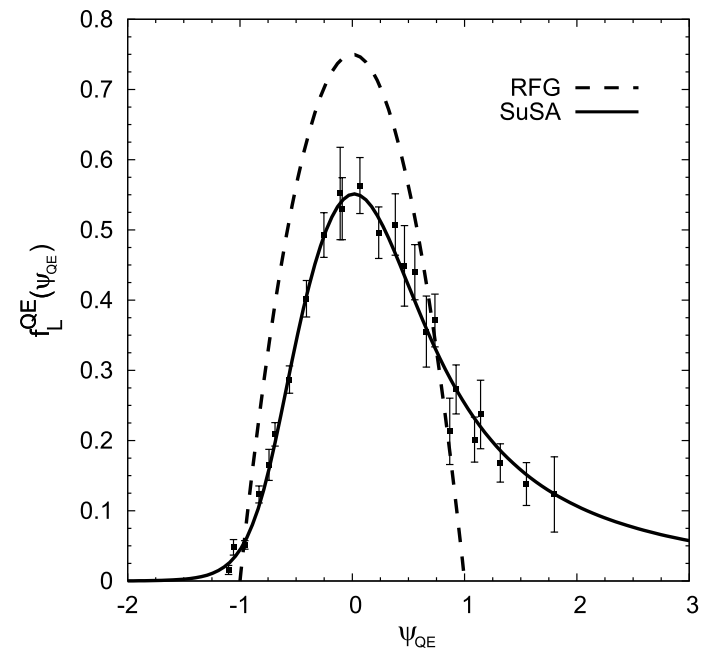

Figure 1. Averaged experimental $f_{L}^{\mathrm{QE}}\left(\psi_{\mathrm{QE}}\right)$ versus $\psi_{\mathrm{QE}}$ in the quasielastic region together with a phenomenological parametrization of the data (solid line). The dashed line shows the scaling function in the RFG model.

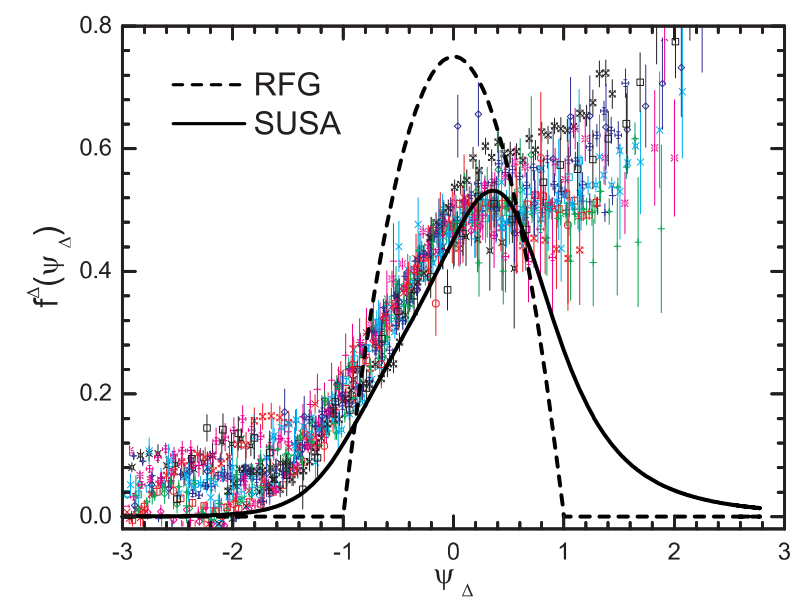

Figure 2. The SuSA scaling function in the $\Delta$-region $f^{\Delta}\left(\psi_{\Delta}\right)$ (solid line) extracted from the world data on electron scattering [3]. The dashed line shows the scaling function $f_{\mathrm{RFG}}^{\Delta}\left(\psi_{\Delta}\right)$ in the RFG model.

We have extended the analysis to $\mathrm{CC}$ pion production cross-section measured at MiniBooNE [13], that from the theoretical point of view can be seen as more challenging. For instance, $\Delta$ properties in the nuclear medium, as well as both coherent and incoherent pion production for the nucleus should be considered in any theoretical approach, while in the SuSA procedure they are included phenomenologically extracted from the electron scattering data. All what is assumed within SuSA approach is the nuclear response to be factorized into a singlenucleon part and a 'nuclear function' accounting for the overall interaction among nucleons. As mentioned before, the SuSA assumptions have been tested against a great deal of electronnucleus scattering data with fair success. The factorization assumption allows one to apply the same nuclear responses derived from electron scattering to neutrino-induced reactions, with a mere use of the adequate single-nucleon terms for this case. To show the importance of nuclear interaction effects as predicted within SuSA, as a reference, we also show results obtained within the RFG, with no interactions among nucleons, for which the scaling function in the $\Delta$-domain is simply given as $f_{\mathrm{RFG}}^{\Delta}\left(\psi_{\Delta}\right)=\frac{3}{4}\left(1-\psi_{\Delta}^{2}\right) \theta\left(1-\psi_{\Delta}^{2}\right)$ with $\psi_{\Delta}$ the dimensionless scaling variable extracted from the RFG analysis that incorporates the typical momentum scale for the selected nucleus $[3,14]$. In figure 2 we compare the $\Delta$-region SuSA [3] and RFG scaling functions, which we use in our study.

\subsection{Charged-current neutrino-nucleus cross section}

We follow the description of the formalism concerning the CC (anti)neutrino-nucleus cross section given in Ref. [3]. The CC neutrino cross section in the target laboratory frame is given in the form:

$$
\left[\frac{d^{2} \sigma}{d \Omega d k^{\prime}}\right]_{\chi} \equiv \sigma_{0} \mathcal{F}_{\chi}^{2}
$$


where $\chi=+$ for neutrino-induced reaction $\left(e . g ., \nu_{\ell}+n \rightarrow \ell^{-}+p\right.$, where $\left.\ell=e, \mu, \tau\right)$ and $\chi=-$ for antineutrino-induced reactions $\left(e . g ., \bar{\nu}_{\ell}+p \rightarrow \ell^{+}+n\right)$,

$$
\sigma_{0} \equiv \frac{\left(G \cos \theta_{c}\right)^{2}}{2 \pi^{2}}\left[k^{\prime} \cos \tilde{\theta} / 2\right]^{2},
$$

$G=1.16639 \times 10^{-5} \mathrm{GeV}^{-2}$ being the Fermi constant, $\theta_{c}$ being the Cabibbo angle $\left(\cos \theta_{c}=\right.$ $0.9741)$, and

$$
\begin{gathered}
\tan ^{2} \tilde{\theta} / 2 \equiv \frac{|Q|^{2}}{v_{0}}, \\
v_{0} \equiv\left(\epsilon+\epsilon^{\prime}\right)^{2}-q^{2}=4 \epsilon \epsilon^{\prime}-|Q|^{2} .
\end{gathered}
$$

In Eqs. (1)-(4) $\Omega, k^{\prime}$ and $\epsilon^{\prime}$ are the scattering angle, momentum and energy of the outgoing lepton. The quantitiy $\mathcal{F}_{\chi}^{2}$ in Eq. (1) depends on the nuclear structure and it is presented in Ref. [3] as a generalized Rosenbluth decomposition having charge-charge, charge-longitudinal, longitudinal-longitudinal, and two types of transverse responses $(R$ 's). These nuclear response functions are expressed in terms of the nuclear tensor $W^{\mu \nu}$ in the QE (as well as in the $\Delta$-region) by means of its relationships with the scaling functions from the particular model used.

\section{3. $\pi^{+}$production in the MiniBooNE experiment}

We present RFG and SuSA predictions for the double-differential cross section for CC neutrinoinduced $\pi^{+}$production (from $\Delta$ resonance region) on $\mathrm{CH}_{2}$ averaged over the neutrino flux $\Phi\left(\epsilon_{\nu}\right)$, namely

$$
\frac{d^{2} \sigma}{d T_{\mu} d \cos \theta}=\frac{1}{\Phi_{\text {tot }}} \int\left[\frac{d^{2} \sigma}{d T_{\mu} d \cos \theta}\right]_{\epsilon_{\nu}} \Phi\left(\epsilon_{\nu}\right) d \epsilon_{\nu}
$$

where $T_{\mu}$ and $\theta$ are correspondingly the kinetic energy and scattering angle of the outgoing muon, $\epsilon_{\nu}$ is the neutrino energy and $\Phi_{\text {tot }}$ is the total integrated $\nu_{\mu}$ flux factor for the MiniBooNE experiment [1]. The nuclear response functions in $\Delta$-region are expressed in terms of the nuclear tensor $W^{\mu \nu}$ in the corresponding region. The basic expressions used to calculate the singlenucleon cross sections are given in [3]. These involve the leptonic and hadronic tensors as well as the response and structure functions for single nucleons. A convenient parametrization of the single-nucleon $W^{+} n \rightarrow \Delta^{+}$vertex is given in terms of eight form-factors: four vector $\left(C_{3,4,5,6}^{V}\right)$ and four axial $\left(C_{3,4,5,6}^{A}\right)$ ones. Vector form factors have been determined from the analysis of photo and electro-production data, mostly on a deuteron target. Among the axial form factors, the most important contribution comes from $C_{5}^{A}$. The factor $C_{6}^{A}$, whose contribution to the differential cross section vanishes for massless leptons, can be related to $C_{5}^{A}$ by PCAC. Since there are no other theoretical constraints for $C_{3,4,5}^{A}\left(q^{2}\right)$, they have to be fitted to data. We use two different parameterizations: the one given in [15] where deuteron effects were evaluated (authors estimated that the latter reduce the cross section by 10\%), denoted as "PR1", and the one from [16], called "PR2".

With these ingredients, we evaluate the cross section for $\mathrm{CC} \Delta^{++}$and $\Delta^{+}$production on proton and neutron, respectively. Once produced, the $\Delta$ decays into $\pi N$ pairs. For the amplitudes $\mathcal{A}$ of pion production the following isospin decomposition applies: $\mathcal{A}\left(\nu_{l} p \rightarrow\right.$ $\left.l^{-} p \pi^{+}\right)=\mathcal{A}_{3}, \mathcal{A}\left(\nu_{l} n \rightarrow l^{-} n \pi^{+}\right)=\frac{1}{3} \mathcal{A}_{3}+\frac{2 \sqrt{2}}{3} \mathcal{A}_{1}, \mathcal{A}\left(\nu_{l} n \rightarrow l^{-} p \pi^{0}\right)=-\frac{\sqrt{2}}{3} \mathcal{A}_{3}+\frac{2}{3} \mathcal{A}_{1}$, with $\mathcal{A}_{3}$ being the amplitude for the isospin $3 / 2$ state of the $\pi N$ system, predominantly $\Delta$, and $\mathcal{A}_{1}$ the amplitude for the isospin $1 / 2$ state that is not considered here.

The double-differential cross section for CC neutrino-induced $\pi^{+}$production averaged over the neutrino energy flux as a function of the muon kinetic energy $T_{\mu}$ is presented in figure 3 . Each panel corresponds to a bin of $\cos \theta$. PR1 and PR2 parametrizations have been considered. Results with the PR1 parameterization are about $5 \%$ higher, that is a measure of the degree 

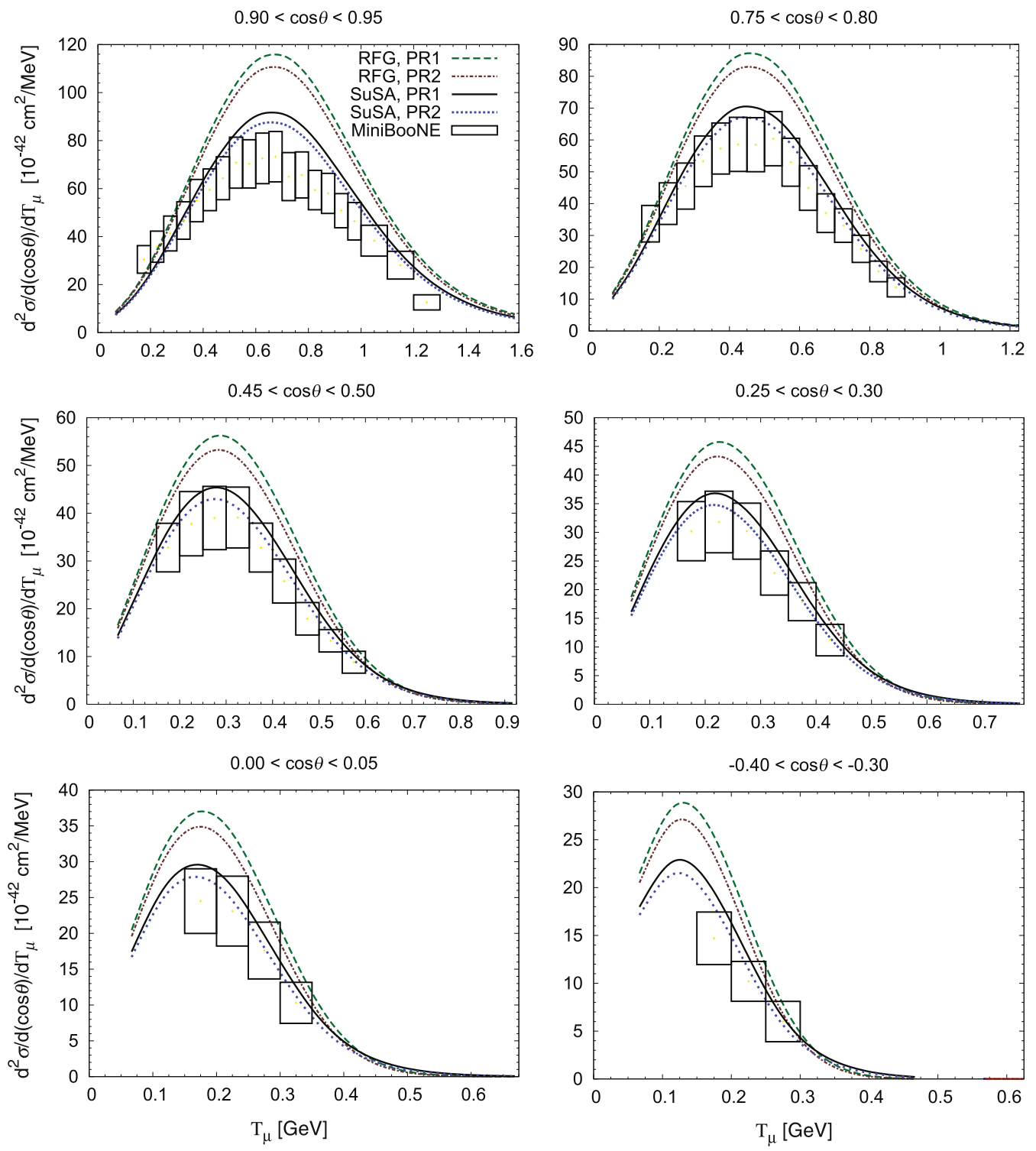

Figure 3. (Color online) The double-differential cross section averaged over the neutrino energy flux as a function of the muon kinetic energy $T_{\mu}$ obtained by SuSA and RFG $\Delta$-region scaling functions. In each subfigure the results have been averaged over the corresponding angular bin of $\cos \theta$. For vector and axial form-factors two parameterizations, "PR1" [15] and "PR2" [16], are used.

of uncertainty that we expect from the choice of the single-nucleon response for this reaction. We compare the predictions of SuSA and RFG with the MiniBooNE data [1]. Here we show that SuSA predictions are in good agreement with the MiniBooNE experimental data for $\pi^{+}$ cross-section in the case of the flux averaged data.

\subsection{Inclusive charged-current neutrino cross sections in the T2K experiment}

In figure 4 we show the $\mathrm{CC}$ inclusive $\nu_{\mu}-{ }^{12} \mathrm{C}$ double-differential cross section per nucleon versus the muon momentum, $p_{\mu}$, for different angular bins, folded with the T2K flux. The QE curve (dashed line) corresponds to the results obtained using SuSA scaling function in the QE-region [3] 

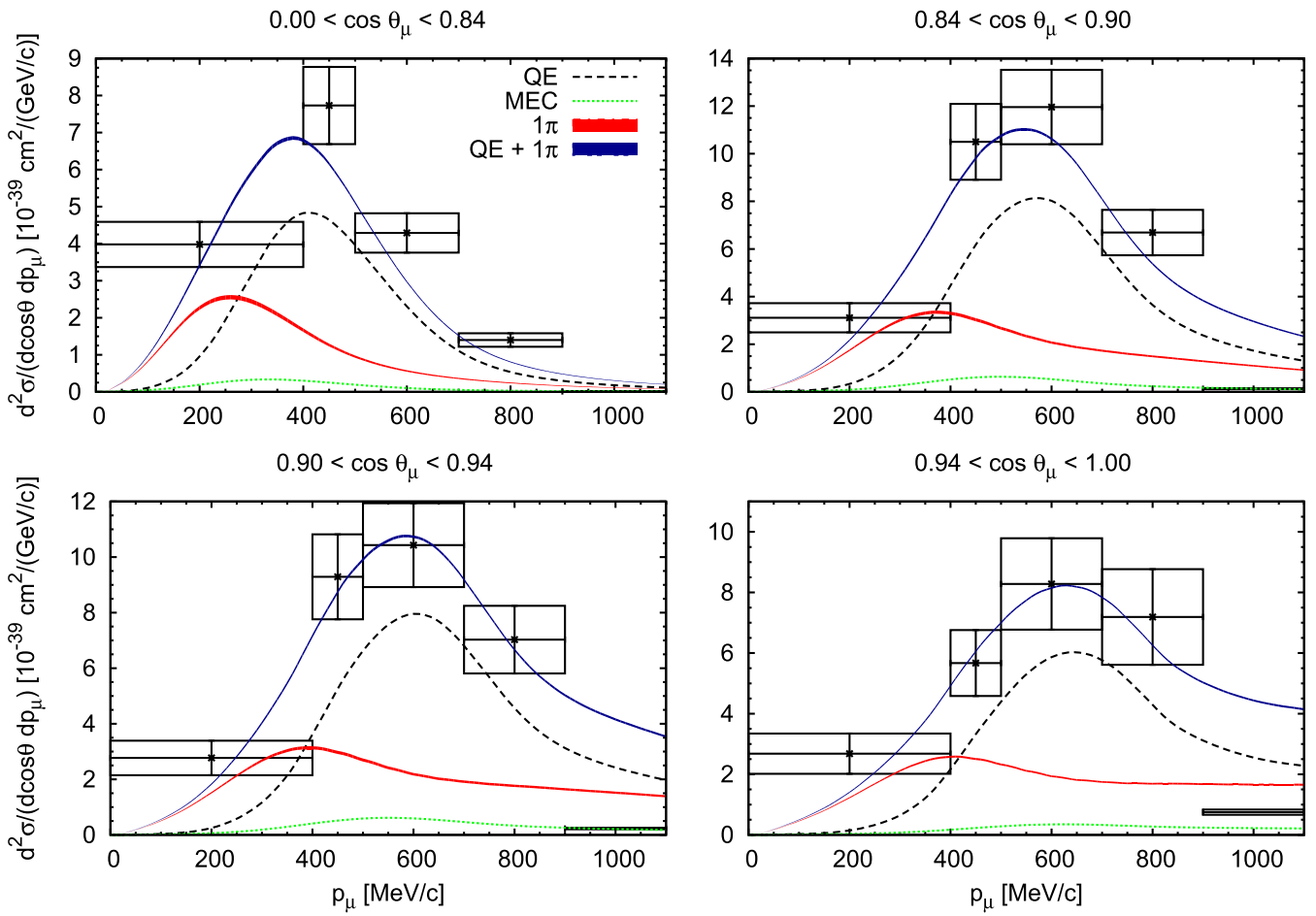

Figure 4. The CC inclusive T2K flux-folded $\nu_{\mu^{-}}{ }^{12} \mathrm{C}$ double-differential cross section per nucleon evaluated using SuSA scaling functions in the QE region [QE] and in the $\Delta$-region $[1 \pi]$ is displayed as a function of the muon momentum for different bins in the muon angle. The separate contributions of the $\mathrm{QE}, 1 \pi$, and vector $2 \mathrm{p} 2 \mathrm{~h}$ MEC are displayed. The data are from [2].

and Pauli blocking effects in the scaling function introduced in Ref. [17]. The standard value of the axial mass $M_{A}=1.03 \mathrm{GeV}$ is used in the $\mathrm{QE}$ calculations. The resonant pion production curve $(1 \pi)$ is derived with the SuSA scaling function in the $\Delta$-region $f^{\Delta}\left(\psi_{\Delta}\right)$ (figure 2). The band corresponds to the two different parametrizations, PR1 and PR2, described in the previous Section.

As first noticed in [3] and studied in more depth in [12], sizeable deviations $(10-15 \%)$ from scaling are observed in the region where the QE and $\Delta$ responses overlap. In this region effects stemming from correlations and $2 \mathrm{p} 2 \mathrm{~h}$ MEC can play an important role and they cannot be reproduced by models that assume impulsive, quasifree scattering on bound nucleons. Therefore these effects must be added to the QE and $\Delta$ scaling functions. In figure 4 the MEC curve (dotted line) corresponds to the fully relativistic calculation of $2 \mathrm{p} 2 \mathrm{~h}$ excitations induced by pionic vector two-body current of [5] and parameterized in [4].

We observe that the model yields good agreement with the T2K data. The main contribution in the cross section comes from the QE and pion production mechanisms. On the contrary, MEC play a minor role at these kinematics. It should be noted however that the present model does not include the axial two-body current.

\section{Conclusions}

The SuSA approach provides neutrino-nucleus cross section predictions, based on the observed nuclear response to electron projectile and the universal character of the scaling function. We show that SuSA predictions are in good agreement with the MiniBooNE experimental data for pionic cross section in the case of the flux averaged data. We conclude that the idea of the 
SuSA approach for the QE- and $\Delta$-regions (extracted from electron scattering experiments), when being extended to neutrino processes, proves to be successful in describing $\nu_{\mu}$ inclusive charged-current cross sections. The model also includes $2 \mathrm{p} 2 \mathrm{~h}$ excitations induced by vector meson-exchange currents carried by the pion. Axial-vector MEC contributions have yet to be included, that might be expected to improve the agreement with the $\mathrm{T} 2 \mathrm{~K}$ data.

\section{Acknowledgments}

This work was partially supported by INFN under project MANYBODY, by Spanish DGI and FEDER funds (FIS2011-28738-C02-01, FPA2013-41267), by the Junta de Andalucia, by the Spanish Consolider-Ingenio 2000 program CPAN (CSD2007-00042), by the Campus of Excellence International (CEI) of Moncloa project (Madrid) and Andalucia Tech, by the Bulgarian National Science Fund under contracts Nos. DFNI-T02/19 and DFNI-E02/6, by the Office of Nuclear Physics of the U.S. Department of Energy under Grant Contract Number DE-FG02-94ER40818 (T.W.D.) and by the European Union 7th FP Marie Curie IOF project ELECTROWEAK (O.M.). R.G.J. acknowledges financial help from the Interuniversity Attraction Poles Programme initiated by the Belgian Science Policy Office.

\section{References}

[1] Aguilar-Arevalo A A et al. (MiniBooNE Collaboration) 2011 Phys. Rev. D 83052007

[2] Abe K et al. (T2K Collaboration) 2013 Phys. Rev. D 87092003

[3] Amaro J E et al. 2005 Phys. Rev. C 71015501

[4] Megias G D et al. 2015 Phys. Rev. D 91073004

[5] De Pace A, Nardi M, Alberico W M, Donnelly T W and Molinari A 2003 Nucl. Phys. A 726303

[6] Donnelly T W and Sick I 1999 Phys. Rev. Lett. 823212

[7] Donnelly T W and Sick I 1999 Phys. Rev. C 60065502

[8] Alberico W M et al. 1988 Phys. Rev. C 381801

[9] Barbaro M B, Cenni R, Pace A D, Donnelly T W and Molinari A 1998 Nucl. Phys. A 643137

[10] Jourdan J 1996 Nucl. Phys. A 603117

[11] Barbaro M B, Caballero J A, Donnelly T W, and Maieron C 2004 Phys. Rev. C 69035502

[12] Maieron C et al. 2009 Phys. Rev. C 80035504

[13] Ivanov M V et al. 2012 Phys. Lett. B $\mathbf{7 1 1} 178$

[14] Maieron C, Donnelly T W and Sick I 2002 Phys. Rev. C 65025502

[15] Alvarez-Ruso L, Singh S K and Vacas M J V 1999 Phys. Rev. C 593386

[16] Paschos E A, Yu J Y and Sakuda M 2004 Phys. Rev. D 69014013

[17] Megias G D et al. 2014 Phys. Rev. D 89093002 